\title{
Comparación de estadios larvales de Helicoverpa zea (Boddie) (Lepidoptera: Noctuidae) mediante longitud de cápsula cefálica y distancia entre setas frontales
}

\author{
Comparative differentiation of instars in Helicoverpa zea (Boddie) \\ using cephalic capsule length and distance between frontal setae
}

\author{
Alfredo Zúñiga ${ }^{1}$, Andrés Angulo ${ }^{2}$, Ramón Rebolledo ${ }^{3}$, María E. Navarro ${ }^{2}$
}

\begin{abstract}
RESUMEN
El gusano del maíz Helicoverpa zea es de una importancia agrícola relevante debido al daño que provoca en los cultivos de maíz y afines, situación que está en directa relación con determinados estadios de su ciclo larval. Fueron colectadas larvas en tres regiones de Chile; norte en Arica, centro en San Clemente y en el sur correspondiendo a Angol, para determinar las diferencias en los estadios larvales por medio del ancho de la cápsula cefálica y la distancia entre setas frontales. Los resultados acusaron diferencias estadísticas significativas entre las larvas colectadas de las regiones de Chile y determinaron que la distancia de las setas cefálicas es una variable mas precisa en la diferenciación de los estadios larvales en relación al ancho de cápsula cefálica.
\end{abstract}

Palabras clave: cápsulas cefálicas, setas cefálicas, latitudes, Helicoverpa zea.

\begin{abstract}
The corn earworm Heliothis zea has relevant agricultural importance because of the damage it causes in corn and other farms, in direct relation to determined larval instars; effective control will be related to the knowledge of the larval biology. Larvae from northern, central and southern Chile were obtained to establish comparisons for the definition of a specific instar measuring width of cephalic capsule and distance between frontal setae (DFS); these structures present a progressive increase in length proportional to the specific instar. The results indicated significant differences among latitudes and that DFS is a better specific instar predictor than the width of the head capsule. The environmental variables that could be implied in the conditioning of lengths by latitudes are discussed as a projection.
\end{abstract}

Key words: Heliothis zea, instars, width of cephalic capsule, distance between frontal setae, Dyar's rule.

\section{Introducción}

El gusano del maíz Helicoverpa zea es una plaga común en los cultivos agrícolas, comprendiendo un gran número de plantas que sirven como hospederas (Angulo et al., 1990), siendo de principal importancia su daño sobre el maíz, el cual puede variar desde un $11 \%$ a un $50 \%$ en los casos más graves (Artigas, 1994). Este efecto es ocasionado en su estado de larva, la cual emerge desde los estigmas hacia el interior de la mazorca, donde empieza a atacar los granos de ésta mientras están suaves, permitiendo la entrada de diversos agentes que finalizan con el deterioro de la mazorca, siendo de esta manera su mayor agente dañino (Artigas, 1994).
El estado larval varía de cinco a siete mudas (Hardwick, 1965), en las cuales es iniciada la acción fitófaga sobre la mazorca, principalmente a partir del segundo estadio, terminando su acción voraz cuando inicia la pupación (Artigas, 1994). Por esta razón es necesario realizar una delimitación morfométrica de cada estadio larval que indique el momento preciso donde deben tomarse medidas en el control de esta plaga. Para este caso la regla de Dyar (1892) sería útil porque establece una relación proporcional entre la longitud de las partes esclerotizadas de un estadio larval y el posterior, siguiendo una razón de tipo geométrica, estableciendo así un modelo predictivo para determinar estadios larvales de cualquier especie analizada.

\footnotetext{
Laboratorio de Ecología Aplicada, Universidad Católica de Temuco.

2 Departamento de Zoología, Facultad de Ciencias Oceanográficas y Naturales, Universidad de Concepción.

3 Facultad de Ciencias Agropecuarias y Forestales, Universidad de La Frontera.

Correo electrónico:zundusicyon@gmail.com
}

Fecha de Recepción: 01 Septiembre, 2008.

Fecha de Aceptación: 28 Febrero, 2011. 
Esta regla se ha aplicado en forma extensa a diversas especies con importancia económica (Beeche et al., 1987; Iraira et al., 2000; Centella et al., 1996), presentando como principal referente la longitud de la cápsula cefálica. Sin embargo, esta estructura presenta limitaciones como consecuencia de su variabilidad, haciendo por consiguiente imprecisa la medición. Un método más adecuado para refinar estos resultados es presentado por Podoler y Klein (1978), quienes establecen una extensión de la regla de Dyar a través de la utilización de las setas frontales como indicador del estado del desarrollo larval. En consecuencia, el objetivo de este trabajo es comparar dos métodos para determinar los estadios larvales de Helicoverpa zea a partir de mediciones del ancho de la cápsula cefálica y distancia entre las setas frontales.

\section{Material y Métodos}

\section{Colecta de material biológico}

Se dispuso de larvas de distintas latitudes de Chile (Norte, Centro, Sur) (Figura 1), las cuales tuvieron la siguiente procedencia y número: Arica, Región de Arica y Parinacota (18 28' S-70 $\left.18^{\circ} \mathrm{W}\right)$. 58 larvas; San Clemente, Región del Maule $\left(35^{\circ}\right.$ 32'S-71 ${ }^{\circ}$ 03'W). 54 larvas; Angol, Región de La Araucanía ( $\left.37^{\circ} 80^{\prime} \mathrm{S}-72^{\circ} 71^{\prime} \mathrm{W}\right)$. 78 larvas.

Estas localidades presentan diferencias en base a las condiciones de los cultivos de maíz (Faiguenbaum, 1987), así como las temperaturas promedio de cada una de ellas (Di Castri y Hajek, 1976).

\section{Preparación de las larvas}

Sólo las cápsulas cefálicas fueron incluidas en $\mathrm{KOH} 10 \%$ para la disolución de los tejidos y grasas, de esta forma las estructuras esclerotizadas se aclararon facilitando la visualización y operatividad en microscopio (Gil et al., 2006).

Las medidas del ancho de las cápsulas cefálicas y la distancia de las setas frontales se realizaron después de ser montadas las estructuras en un portaobjetos. Posteriormente fue realizada la observación de éstas con ayuda de microscopio óptico marca Zeiss, el cual permitió realizar las mediciones por medio de líneas absolutas que a su vez fueron transformadas a escala milimétrica por medio del factor correspondiente al objetivo utilizado.

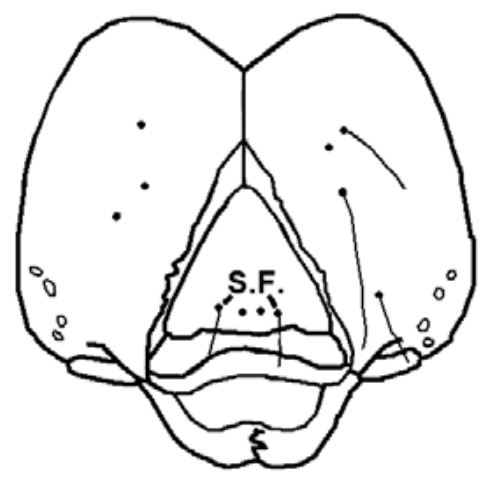

Figura 1. Representación esquemática de la cápsula cefálica de Helicoverpa zea (Angulo \& Weigert, 1975). S.F. $=$ Setas frontales.

Se procedió a la representación gráfica de los datos en las dos variables, los cuales al disponerse en forma polimodal se reconocieron como rango de longitudes para cada estadio. Se recogieron estos datos y se sistematizaron de acuerdo a lo desarrollado por Flores et al. (2005). La precisión en base a los estadios tuvo como parámetro de definición el error estándar que presentó cada una de las variables, de modo tal que un menor coeficiente de variación respecto a la media indicó el método más adecuado para diferenciar los estadios de la especie.

La falta de material de larvas de los primeros estadios para su representación en el modelo, debido a que éstos se inician en los estigmas del maíz, se infirieron a partir de las proporciones obtenidas a partir de los estadios superiores, tomando de esta manera un carácter referencial.

\section{Resultados y Discusión}

Los resultados obtenidos de las mediciones en ambos caracteres se presentan en las tablas I-II y III. Éstos presentaron variaciones en el número de estadios larvales. Esta situación puede argumentarse debido en las diferentes condiciones de los cultivos de maíz en las áreas de estudio (Faiguenbaum, 1987), lo que determinaría las diferencias métricas en cada caso. De la misma manera, la diferencia de estadios larvares encontrados de Helicoverpa zea respecto a lo mencionado en la literatura (Artigas, 1994) refleja el efecto que las variables medioambientales ejercen sobre esta situación (Daly, 1985), como el alimento disponible (Rebolledo y Arroyo, 1995) y la temperatura (Podoler y Klein, 1978), los cuales en consideración con las distintas latitudes muestreadas tienden a afectar el número de estadios 
larvales. Otras situaciones anexas a los cultivos, como la resistencia adquirida hacia la plaga por parte de los nuevos cultivares de maíz (Giustiolin et al., 1994) actuarían alargando el número de estadios larvales, situación que limitaría la precisión metodológica para delimitar los mismos (Parra y Haddad, 1989).

Al comparar el grado de precisión, establecido como el error estándar resultante de la medición de ambas estructuras, éste resultó ser mayor en el caso de la distancia entre setas frontales, situación que se presentó en las tres localidades evaluadas. De la misma manera, al comparar el coeficiente de variación en esta estructura tiende a ser menor en las mismas circunstancias, habiendo sólo mínimas excepciones que no alteran esta tendencia.

Las setas cefálicas, al tener poca exposición al exterior debido a que es una estructura contenida en la cápsula, tienen una baja probabilidad de verse afectadas por efectos mecánicos y por consiguiente tienden a reflejar de una forma más precisa el incremento de longitud entre estadios larvales respecto al ancho de cápsula cefálica, la cual, al presentar un mayor coeficiente de variación, pone de manifiesto que esta estructura puede distorsionar la precisión de longitudes para determinar los estadios larvales,

Tabla I. Estadios larvales de Helicoverpa zea de acuerdo al ancho de cápsula cefálica y la distancia entre setas frontales obtenidos de localidades de la Región de Arica y Parinacota.

\begin{tabular}{|c|c|c|c|c|c|c|c|c|c|}
\hline $\begin{array}{l}\text { Estadio } \\
\text { larval }\end{array}$ & $\mathrm{N}$ & $\begin{array}{c}\text { Media ancho } \\
\text { cápsula } \\
\text { cefálica }\end{array}$ & $\begin{array}{c}\text { Error } \\
\text { estándar }\end{array}$ & $\begin{array}{l}\text { Coeficiente } \\
\text { de variación }\end{array}$ & $\begin{array}{c}\text { Coeficiente } \\
\text { Dyar }\end{array}$ & $\begin{array}{l}\text { Media dis- } \\
\text { tancia setas } \\
\text { frontales }\end{array}$ & $\begin{array}{c}\text { Error } \\
\text { estándar }\end{array}$ & $\begin{array}{c}\text { Coeficiente } \\
\text { de variación }\end{array}$ & $\begin{array}{c}\text { Coeficiente } \\
\text { Dyar }\end{array}$ \\
\hline I & - & 0.314 & & & 1.50 & 0.044 & & & \\
\hline II & - & 0.472 & & & 1.50 & 0.066 & & & 1.47 \\
\hline III & - & 0.708 & & & 1.50 & 0.095 & & & 1.46 \\
\hline IV & 6 & 1.063 & 0.032 & 8.77 & 1.46 & 0.191 & 0.0010 & 1.05 & 1.50 \\
\hline V & 8 & 1.553 & 0.11 & 11.57 & 1.53 & 0.287 & 0.0080 & 4.51 & 1.48 \\
\hline VI & 18 & 2.390 & 0.038 & 6.77 & 1.54 & 0.426 & 0.0060 & 8.68 & 1.37 \\
\hline VII & 26 & 3.33 & 0.025 & 3.89 & 1.38 & 0.650 & 0.0013 & 10.30 & 1.48 \\
\hline
\end{tabular}

Tabla II. Estadios larvales de Helicoverpa zea de acuerdo al ancho de cápsula cefálica y la distancia entre setas frontales obtenidos de localidades de la Región del Maule.

\begin{tabular}{|c|c|c|c|c|c|c|c|c|c|}
\hline $\begin{array}{l}\text { Estadio } \\
\text { larval }\end{array}$ & $\mathrm{N}$ & $\begin{array}{l}\text { Media ancho } \\
\text { cápsula } \\
\text { cefálica }\end{array}$ & $\begin{array}{c}\text { Error } \\
\text { estándar }\end{array}$ & $\begin{array}{l}\text { Coeficiente } \\
\text { de variación }\end{array}$ & $\begin{array}{l}\text { Coeficiente } \\
\text { de } \\
\text { crecimiento }\end{array}$ & $\begin{array}{c}\text { Media } \\
\text { distancia } \\
\text { intersetal }\end{array}$ & $\begin{array}{c}\text { Error } \\
\text { estándar }\end{array}$ & $\begin{array}{l}\text { Coeficiente } \\
\text { de variación }\end{array}$ & $\begin{array}{l}\text { Coeficiente } \\
\text { de } \\
\text { crecimiento }\end{array}$ \\
\hline I & - & $0.320(*)$ & & & & $0.050(*)$ & & & \\
\hline II & - & $0.480(*)$ & & & 1.50 & $0.075(*)$ & & & 1.50 \\
\hline III & - & $0.720(*)$ & & & 1.50 & $0.113(*)$ & & & 1.50 \\
\hline IV & - & $1.070(*)$ & & & 1.43 & $0.169(*)$ & & & 1.45 \\
\hline $\mathrm{V}$ & 13 & 1.554 & 0.045 & 23.10 & 1.45 & 0.246 & 0.005 & 8.53 & 1.42 \\
\hline VI & 20 & 2.217 & 0.045 & 9.02 & 1.42 & 0.394 & 0.012 & 14.35 & 1.60 \\
\hline VII & 31 & 3.020 & 0.014 & 2.31 & 1.36 & 0.550 & 0.0034 & 3.45 & 1.39 \\
\hline
\end{tabular}

Tabla III. Estadios larvales de Helicoverpa zea de acuerdo al ancho de cápsula cefálica y la distancia entre setas frontales obtenidos de localidades de la Región de La Araucanía.

\begin{tabular}{|c|c|c|c|c|c|c|c|c|c|}
\hline $\begin{array}{l}\text { Estadio } \\
\text { larval }\end{array}$ & $\mathrm{N}$ & $\begin{array}{l}\text { Media ancho } \\
\text { cápsula } \\
\text { cefálica }\end{array}$ & $\begin{array}{c}\text { Error } \\
\text { estándar }\end{array}$ & $\begin{array}{l}\text { Coeficiente } \\
\text { de variación }\end{array}$ & $\begin{array}{c}\text { Coeficiente } \\
\text { Dyar }\end{array}$ & $\begin{array}{c}\text { Media } \\
\text { distancia } \\
\text { intersetal }\end{array}$ & $\begin{array}{c}\text { Error } \\
\text { estándar }\end{array}$ & $\begin{array}{l}\text { Coeficiente } \\
\text { de variación }\end{array}$ & $\begin{array}{c}\text { Coeficiente } \\
\text { Dyar }\end{array}$ \\
\hline I & - & $0.300(*)$ & & & & $0.054(*)$ & & & \\
\hline II & - & $0.440(*)$ & & & 1.45 & $0.079(*)$ & & & 1.45 \\
\hline III & - & $0.660(*)$ & & & 1.50 & $0.115(*)$ & & & 1.45 \\
\hline IV & 8 & 1.040 & 0.064 & 17.59 & 1.57 & 0.167 & 0.009 & 16.16 & 1.45 \\
\hline V & 13 & 1.578 & 0.037 & 8.49 & 1.51 & 0.286 & 0.009 & 12.23 & 1.71 \\
\hline VI & 28 & 2.267 & 0.048 & 11.33 & 1.43 & 0.429 & 0.008 & 10.02 & 1.50 \\
\hline VII & 29 & 3.297 & 0.040 & 6.64 & 1.45 & 0.630 & 0.011 & 9.52 & 1.46 \\
\hline
\end{tabular}


así como complicaciones a consecuencia de su manipulación para su observación (Flores et al., 2005). Asimismo, la menor precisión del ancho de cápsula cefálica ha sido puesta de manifiesto debido a la sobreposición de longitudes que presenta entre estadios, lo que no sucede con la distancia intersetal (Centella et al., 1996), lo que no lo validaría como un criterio muy preciso para la definición de estos estadios.

\section{Conclusiones}

Las larvas de Helicoverpa zea presentaron variaciones en el número de sus estadios larvales de acuerdo a las localidades en donde se desarrollaron los estudios. De la misma manera, las setas cefálicas de éstas exhibieron una mayor precisión para determinar un estadio larval específico que las cápsulas cefálicas, debido a la menor variación inherente de estos caracteres.

\section{Agradecimientos}

Al proyecto FIP-05 de la Dirección de Investigación de la Universidad Católica de Temuco, y a Héctor Vargas por su ayuda en la colecta de material.

\section{Literatura Citada}

Angulo, A.; Jana-Sáenz, C.; Parra, L.E.; Castillo, E. 1990 Lista de Lepidópteros Noctuidos asociados a algunos cultivos en Chile (Lepidoptera:Noctuidae): Status sistemático actual. Gayana Zoología 54 (1-2): 51-61.

Artigas, J.N.

1994 Entomología Agrícola. Ediciones Universidad de Concepción. Vol. 2. 848 p.

Beeche, M.; Parra, L.; Cerda, L.

1987 Descripción de los estados preimaginales y desarrollo biológico de Omaguacua longibursae Parra y Beeche, 1986 (Lepidoptera: Geometridae). Boletín de la Sociedad de Biología de Concepción, Chile. Tomo 58, 19-29 pp.

Centella, C.; Urzúa, C.; Caro, J.; Jerez, V.

1996 Descripción etaria para los estadios larvarios de Rhyacionia buoliana (Lepidoptera:Tortricidae). Bosque 17 (1): 91-96.

Daly, $\mathrm{H}$.

1985 Insect morphometrics. Ann. Rev. Entomol. 30: 415-438.

Di Castri, F.; Hajek, E.

1976 Bioclimatología de Chile. Ediciones Universidad Católica de Chile, Santiago. 127 pp.

Dyar, H.G.

1890 The number of molts of Lepidopterous larvae. Psyche, Camb. 5: 420-422.

Faiguenbaum, $\mathrm{H}$.

1987 Producción de cultivos en Chile. Cereales, Leguminosas e Industriales. Publicitaria Torrelodones Ltda. Santiago, Chile. 332 pp.

Flores, L.; Bautista, N.; Martínez, N.; Valdez, J.; Morales, O.; Quiñones, S.
2005 Comparación de dos técnicas de medición de cápsulas cefálicas para separar estadios larvales de Copitarsia incommoda (Walker) (Lepidoptera:Noctuidae). Acta Zoológica Mexicana 21 (2): 109-113.

Gil, M.; Garelis, P.A.; Vallenia, E.A.

2006 Hábitos alimenticios de larvas de Polycentropus joergenseni Ulmer, 1909 (Trichoptera: Polycentropodidae) en el Río Grande (San Luis, Argentina). Gayana 70 (2): 206-209.

Giustolin, T. A.; Vendramin, J.D.

1994 Efeito de duas espécies do tomateiro na biología de Scrobipalpuloides absoluta (Meryck). Anais da Sociedade Entomologica do Brasil. V.23, 511-517.

Hardwick, D

1965 The corn earworm complex. Memoirs of the Entomological Society of Canada. 107-116 pp.

Iraira, S.; Rebolledo, R.; Aguilera A.

2000 Aspectos biológicos de la polilla mediterránea de la harina Anagasta kuehniella (Zeller) (Lepidoptera: Pyralidae) criada en polen. Rev. Chilena de Entomología. 27: 79-84

Parra, J.R.P.; Hadad, M. De L. 1989 Determinaçao do Número de Ínstares de Insectos. FEALQ, Piracicaba, 49 pp.

Podoler, H.; Klein, M.

1978 Distance between frontal setae: a new tool for determining caterpillar instars. J. Nat. Hist. 12 (3): 341-347 pp.

Rebolledo, R.; Arroyo, M.

1995 Estudio del comportamiento de Trogoderma granarium Everts (Coleoptera: Dermestidae) en diapausa debida a la ausencia de alimento. Bol. San. Veg. Plagas (España) 21 (3): 319-327. 